\title{
Catalytic Role of Refractory Interstellar Grain Analogs on $\mathrm{H}_{2}$ Formation
}

\author{
Tushar Suhasaria and Vito Mennella* \\ Istituto Nazionale di Astrofisica (INAF)-Osservatorio Astronomico di Capodimonte, Naples, Italy
}

Refractory dust grains have an important role to play in the chemistry of star and planet-forming regions. Their surfaces interact with interstellar gas and act as a catalyst for the formation of simple and complex molecules in space. Several mechanisms have been invoked to explain how molecular hydrogen is formed in reactions on dust grain surfaces in different regions of space. In this article, we give an overview of our understanding of the laboratory experiments, conducted over the last 20 years, that deal with $\mathrm{H}_{2}$ formation on interstellar grain analogs in space simulated conditions.

Keywords: laboratory astrochemistry, dust analogs, molecular hydrogen, surface chemistry, interstellar medium

\section{OPEN ACCESS}

Edited by:

Ryan C. Fortenberry,

University of Mississippi, United States

Reviewed by:

Martin Robert Stewart McCoustra,

Heriot-Watt University,

United Kingdom

Gianfranco Vidali,

Syracuse University, United States

*Correspondence:

Vito Mennella

vito.mennella@inaf.it

Specialty section:

This article was submitted to

Astrochemistry,

a section of the journal

Frontiers in Astronomy and Space

Sciences

Received: 19 January 2021 Accepted: 06 April 2021 Published: 07 May 2021

Citation:

Suhasaria T and Mennella V (2021) Catalytic Role of Refractory Interstellar

Grain Analogs on $\mathrm{H}_{2}$ Formation.

Front. Astron. Space Sci. 8:655883.

doi: 10.3389/fspas.2021.655883

\section{INTRODUCTION}

The interstellar medium (ISM) is the tenuous gas and dust that pervades space between the stars in a galaxy. Interstellar gas is the major component that accounts for $99 \%$ of the interstellar mass, of which $70 \%$ is hydrogen either in atomic or molecular form (Ferriere, 2001). Solid refractory dust, which is made up of either silicates or carbonaceous materials, forms the remaining $1 \%$ of the interstellar mass. Molecular hydrogen $\left(\mathrm{H}_{2}\right)$ is by far the most abundant molecule in the Universe and it plays a crucial role in star formation. It also directly or indirectly contributes to all the reactions involved in the formation of simple and complex molecules in the ISM.

Naturally, a lot of interest lies in the understanding of how $\mathrm{H}$ atoms convert to $\mathrm{H}_{2}$ in different environments of space. In regions of low gas density and temperatures, $\mathrm{H}_{2}$ cannot form efficiently in the gas phase by the association of two $\mathrm{H}$ atoms. Therefore, the need to explore an alternative route of molecule formation becomes crucial to explain the observed abundances. Van de Hulst (1948) was the first to propose a significant role of dust grains in molecule formation. Dust grain surfaces can impart catalytic effect by (i) acting as a site for atoms to rapidly diffuse and react; (ii) acting as the third body to dissipate the recombination energy; (iii) reducing the reaction activation barriers. Based on the rate constant calculations of formation and destruction processes of $\mathrm{H}_{2}$ in interstellar space, it was suggested that recombination of $\mathrm{H}$ atoms on dust grain surface could proceed efficiently between 5 and $20 \mathrm{~K}$ (Gould and Salpeter, 1963). To explore the possibility of extending an efficient recombination of $\mathrm{H}$ atoms on the grain surface beyond $20 \mathrm{~K}$, calculations were made with a more realistic model of interstellar grain having lattice defects or impurity sites rather than regular grain surface (Hollenbach and Salpeter, 1971). The $\mathrm{H}_{2}$ formation rate, calculated for the dust grain catalyzed reaction, was found to be compatible with the observed $\mathrm{H}_{2}$ abundances in diffuse ISM. A large number of experimental studies was also devoted to the understanding of $\mathrm{H}_{2}$ formation. However, their relevance to astrophysical conditions is not easy to derive since these studies were not performed on realistic interstellar grain analog surfaces and conditions encountered in space. For an account of those studies, see Pirronello et al. (2000). It was only in the late 1990s that laboratory experiments were performed on well-characterized interstellar 
grain analogs in simulated space conditions to examine the nuances of gas-grain chemistry relating to $\mathrm{H}_{2}$ formation. For a systematic review on $\mathrm{H}_{2}$ formation on interstellar dust grains, see Vidali (2013) and Wakelam et al. (2017). The aim of the present paper is to outline relevant laboratory experiments that discuss the catalytic role of the refractory dust grains in $\mathrm{H}_{2}$ formation on different grain temperatures found in varied regions of space.

\section{REFRACTORY INTERSTELLAR DUST GRAINS}

Refractory dust grains are primarily formed in the circumstellar envelope of evolved stars during the asymptotic giant branch phase. The chemical composition of the dust is determined by the composition of the evolved stars producing it. There are two main classes of grains whose composition depends on the $\mathrm{C} / \mathrm{O}$ abundance ratio in the stellar atmosphere. The carbonrich outflows $(\mathrm{C} / \mathrm{O}>1)$ lead to the production of carbonaceous grains (e.g., amorphous carbon, silicon carbide, titanium carbide, etc.). In oxygen-rich envelopes $(\mathrm{C} / \mathrm{O}<1)$, silicates (olivine and pyroxene composition) and other metal oxides (e.g., $\mathrm{MgO}, \mathrm{FeO}$, $\mathrm{Fe}_{3} \mathrm{O}_{4}, \mathrm{Al}_{2} \mathrm{O}_{3}$ ) are formed. Other sources of refractory grains are the nova and supernova ejecta and $\mathrm{R}$ Coronae Borealis variable stars.

During the journey of dust grains through the ISM, they interact with cosmic rays, UV photons, atoms which induce many effects such as amorphization, sputtering, and implantation. As the dust traverses through dense and UVshielded molecular clouds, various simple molecules (mainly $\mathrm{H}_{2} \mathrm{O}$ ) accrete on them forming icy mantles. Observational evidence suggest that silicates, carbonaceous grains and water ice mantles are predominantly amorphous in the ISM. According to interstellar dust models, silicate grains are mainly sub-micron in size, while carbon grains range in size from nano to submicron (Draine, 2003; Jones et al., 2013). Krugel (2007) and Draine (2010) study provide a detailed review of the structure and composition of dust.

\section{PREPARATION OF LABORATORY DUST ANALOGS}

Laboratory dust analogs are prepared with the aim to understand how dust grains are formed, destroyed and altered in different astrophysical environments, and how these processes affect their spectral properties. Realistic analogs, which simulate the chemical composition and properties of the cosmic dust, have also been considered to investigate the role of dust grain surfaces for molecule formation. Different methods, such as arc discharge, laser ablation, chemical vapor deposition, flame synthesis, ion sputtering, sol-gel, have been applied in the laboratory for the production of dust analogs. The prepared analogs are thoroughly characterized by different techniques among which wet chemical analysis, scanning electron microscope, energy dispersive x-ray and x-ray diffraction are commonly used. A detailed report on the preparation and characterization of silicate and carbonaceous grain analogs can be found in Colangeli et al. (2003), Henning (2010), and Jäger et al. (2015).

\section{REACTION MECHANISMS BETWEEN ADSORBATE AND SURFACE}

Physisorption and chemisorption are the two types of interactions that are important to consider between an atom and a surface. The former refers to weak and long range van der Waals forces that involve binding energy of the order of tens of $\mathrm{meV}$. In the second case, there are strong and localized chemical bonds with a binding energy of the order of a few $\mathrm{eV}$. Formation of $\mathrm{H}_{2}$ on surfaces can occur through three main mechanisms namely Langmuir-Hinshelwood (LH), Eley-Rideal (ER), and Harris-Kasemo (HK, commonly known as hot atom). In LH mechanism, at first atoms are thermally accommodated on the surface, then they diffuse (via thermal hopping or quantum tunneling) and react to form molecules at the surface temperature. In the other two processes, atoms from the gas phase directly (for ER) or indirectly (for HK) hit other pre-adsorbed atoms on the surface. The HK is considered as an intermediate mechanism between LH and ER.

In the case of $\mathrm{H}_{2}$ formation in space, $\mathrm{H}$ atoms can remain physisorbed on the grain surface until its temperature is low enough to prevent desorption. This happens in the cold interstellar clouds where grain temperatures are typically $<20$ $\mathrm{K}$. However, in these conditions $\mathrm{H}$ atoms are mobile enough to initiate reactions mainly through $\mathrm{LH}$ mechanism. Besides, in warmer environments such as photodissociation regions (PDRs), while physisorption fails to hold $\mathrm{H}$ atoms on the grain surface, chemisorbed $\mathrm{H}$ atoms that remain on the surface take part in formation reaction. In this regard, atoms are immobile and $\mathrm{LH}$ pathway can be fairly ruled out. Atoms from the gas phase with enough energy are needed to overcome the activation barrier to react on the surface, and molecule formation takes place via ER and HK mechanism. In the sub-sections that follow, we describe the $\mathrm{H}_{2}$ formation in laboratory experiments performed at low and high grain surface temperatures.

\section{1. $\mathrm{H}_{2}$ Formation at Low Grain Surface Temperature}

The first set of experiments to investigate $\mathrm{H}_{2}$ formation on a dust grain analog was performed on a natural, polycrystalline olivine sample (Pirronello et al., 1997a,b). Together with the prompt formation of molecular hydrogen during irradiation of the surface by $\mathrm{H}$ atoms, the temperature programmed desorption (TPD) technique was applied to measure the recombination efficiency of $\mathrm{H}$ and $\mathrm{D}$ atoms (possessing low kinetic energies) that were still on the surface for a range of dust grain temperatures $(<20 \mathrm{~K})$ encountered in the ISM (see Figure 1). The HD formation efficiency was found to be lower than the other theoretical model based estimates. The efficiency decreases with increasing sample temperature due to a short residence time of physisorbed H/D atoms at high temperatures. This aspect is also demonstrated by the trend with temperature of the sticking probability of $\mathrm{H}$ atoms on a dust analog surface (see Figure 2). 


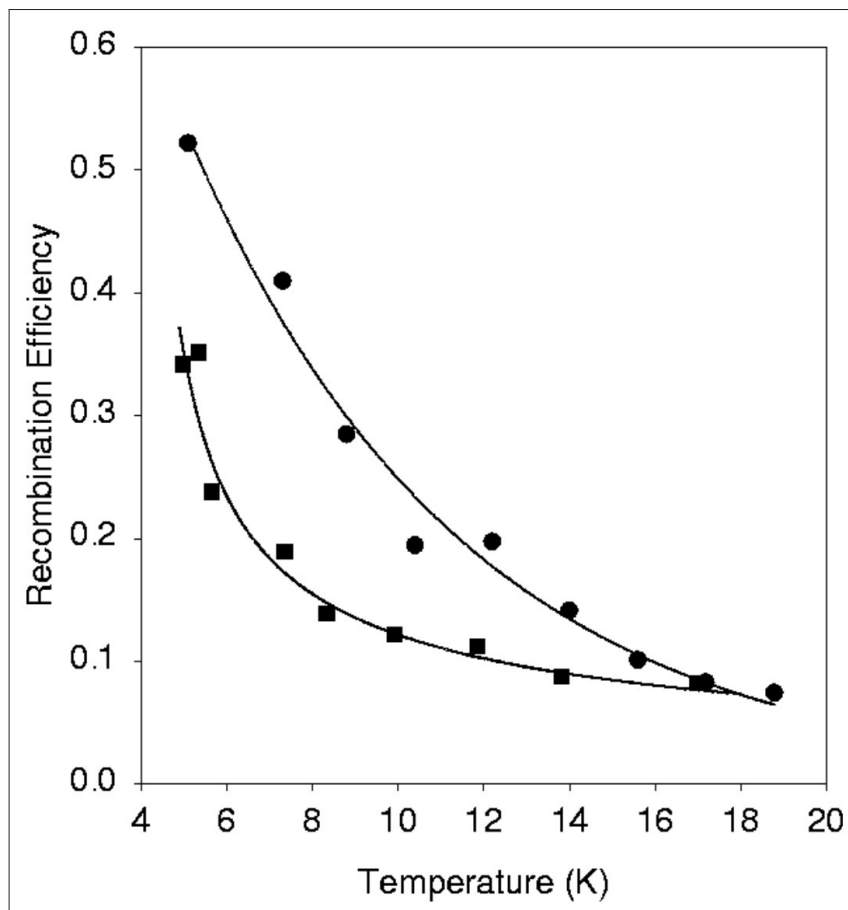

FIGURE 1 | HD recombination efficiency as a function of temperature on a surface of amorphous carbon grains (top, filled circles) and crystalline olivine (bottom, filled squares). Lines are guides to the eye. From Pirronello et al. (1999), reproduced with permission from Astronomy \& Astrophysics, CESO.

HD formation on olivine followed a second order kinetics at low coverages of $\mathrm{H}$ and $\mathrm{D}$ atoms. This means that atoms continued to remain localized on the surface without recombining till the surface temperature was raised. HD formation experiments were also carried out on amorphous carbon grains (prepared using the arc-discharge method) in the experimental conditions identical to the previous set (Pirronello et al., 1999). HD recombination efficiency was found to be higher in amorphous carbon grains than the silicates (Figure 1). The authors attributed this difference to the variation in surface morphology. A rate equation model, reproducing the above experimental data, confirmed that $\mathrm{H}_{2}$ molecules can form efficiently only at low temperatures i.e., in the range $6-10 \mathrm{~K}$ for olivine and $10-20 \mathrm{~K}$ for amorphous carbon grains (Katz et al., 1999). Identical experiments of HD formation, as discussed above, were performed on amorphous olivine with different compositions prepared by laser ablation of mixed oxides used in varying proportions (Perets et al., 2007; Vidali et al., $2007,2009)$. Amorphous silicate grains were found to be a more efficient catalyst for $\mathrm{H}_{2}$ formation than polycrystalline olivine because $\mathrm{H}_{2}$ formation efficiency is extended and shifted to higher grain temperature in the case of amorphous silicates. It was also suggested that $\mathrm{H}_{2}$ molecules thermalize to the grain temperature before desorption. In another study, HD formation on single crystal olivine was found to be less effective than both amorphous and polycrystalline silicates (He et al., 2011). An interesting class of laboratory experiments that concerns $\mathrm{H}_{2}$ formation at low dust grain temperature are reactions on amorphous water ice mantles covering the refractory dust grains. It has been shown that such surfaces at $8 \mathrm{~K}$ possess shallow and deep adsorption sites that allows trapped $\mathrm{H}$ atoms to wait for the arrival of other $\mathrm{H}$ atoms from the gas phase in the low flux conditions of interstellar clouds and facilitate $\mathrm{H}_{2}$ formation (Watanabe et al., 2010). Although $\mathrm{H}_{2}$ formation on interstellar ices has been a topic of much interest, it is beyond the scope of the present article (see, for example, Watanabe and Kouchi (2008) and Hama and Watanabe (2013) for a review on this subject.

The process of $\mathrm{H}_{2}$ formation raises an important concern about the fate of the $4.5 \mathrm{eV}$ energy released during $\mathrm{H}$ atom recombination. In other words, we need to understand how this energy is distributed between a grain surface and the nascent molecule ejecting from the surface. TPD and resonance enhanced multiphoton ionization (REMPI) spectroscopy were simultaneously applied to study energy partitioning as a function of sample temperature during $\mathrm{D}_{2}$ formation on amorphous silicate films (Lemaire et al., 2010). The films were prepared by electron beam evaporation of a San Carlos olivine on a gold coated substrate. The $\mathrm{D}_{2}$ molecules, desorbed promptly just after formation, were detected in high excited states (vibrational level $=4$, rotational level $=2$ ). It was inferred that the morphology of the surface influences the internal energy of the formed molecules. In fact, $\mathrm{D}_{2}$ molecules ejected from the crystalline sample were found to have a lower rotational energy distribution than those desorbed from amorphous samples of varying stoichiometry prepared by laser ablation technique (Gavilan et al., 2014). Similar sort of experiments were also performed on highly oriented pyrolitic graphite (HOPG), an idealized representation of an interstellar grain surface, between 15 and 50 K (Creighan et al., 2006; Islam et al., 2007; Latimer et al., 2008). In these experiments, HD molecules that were leaving the surface upon formation were detected in the vibrationally excited state up to the 7th level. Such investigations are important not only to look for observational signatures during $\mathrm{H}_{2}$ formation in space, but also to develop accurate theoretical models.

\section{2. $\mathrm{H}_{2}$ Formation at High Grain Surface Temperature}

For $\mathrm{H}_{2}$ formation at high dust grain temperatures, reaction mechanisms that involve tightly bound chemisorbed $\mathrm{H}$ atoms on graphite and highly defected carbon/ silicate surface were theoretically proposed (Cazaux and Tielens, 2004; Cuppen and Herbst, 2005). Experimentally, adsorption behavior of H (D) atoms on HOPG was investigated and recombinative desorption of $\mathrm{H}_{2}\left(\mathrm{D}_{2}\right)$ showed a double peak in the TPD spectra (Zecho et al., 2002a). The desorption activation energy of $\mathrm{H}$ (D) on the surface was estimated to be $\sim 0.6(0.9) \mathrm{eV}$. Scanning tunneling microscopy (STM) images revealed that $\mathrm{H}_{2}$ recombination is possible from two hydrogen dimer states on HOPG (Hornekær et al., 2006b). These dimer states were identified as the origin of the unusual double peak in TPD traces for hydrogen on graphite. Moreover, activation barriers of $\sim 1.2$ and $\sim 1.6 \mathrm{eV}$ were calculated for $\mathrm{H}_{2}$ formation. STM images further revealed that $\mathrm{H}$ atoms have a preferential sticking on HOPG on those sites where an $\mathrm{H}$ atom is already pre-adsorbed, resulting in the formation of 
large clusters that favor $\mathrm{H}_{2}$ formation (Hornekær et al., 2006a). In another study, HD formation via ER or HK abstraction was investigated after exposure of $\mathrm{H}$ atoms with kinetic energies of 2,000 K on deuterated HOPG at $150 \mathrm{~K}$ (Zecho et al., 2002b). Depending on the coverage of the chemisorbed $\mathrm{D}$ atoms on the surface, the abstraction cross-section varied between 4 and 17 $\AA^{2}$. Concerning the partitioning of $4.5 \mathrm{eV}$, the kinetic energy of $\mathrm{H}_{2} / \mathrm{D}_{2}$ molecules formed by laser assisted associative desorption of H/D atoms on HOPG at room temperature showed a broad distribution with a peak at $\sim 1.3 \mathrm{eV}$ (Baouche et al., 2006).

$\mathrm{H}_{2}$ formation on amorphous carbonaceous surface with aliphatic $\mathrm{C}-\mathrm{H}$ bonds was initially ignored since the binding energies of $\mathrm{H}$ atoms were considered very high $(\sim 4 \mathrm{eV})$. Mennella (2006) has experimentally estimated an activation barrier of $6 \mathrm{meV}(70 \mathrm{~K})$ for $\mathrm{H}$ atoms to form the aliphatic $\mathrm{CH}_{2,3}$ groups on hydrogen free nano-sized amorphous carbon grains. The barrier is much lower than the typical values of few tenths of an $\mathrm{eV}$ derived for $\mathrm{H}$ atoms on graphite (Hornekær et al., 2006b). On such hydrogenated amorphous carbon surface, HD formation has been observed for a range of surface temperatures 13-300 K, and for impinging D atoms between 80 and 300 $\mathrm{K}$ through ER mechanism (Mennella, 2008). At the aliphatic $\mathrm{CH}_{2,3}$ groups, $\mathrm{H} / \mathrm{D}$ exchange occurs through a two-step reaction sequence: (1) dehydrogenation via $\mathrm{H}$ atom abstraction with the formation of an HD molecule and (2) deuteration with the addition of a D atom. Figure 3 (red curve a) depicts the intensity decrease of the $\mathrm{C}-\mathrm{H}$ stretching feature of aliphatic $\mathrm{CH}_{2,3}$ groups and the simultaneous increase of the corresponding aliphatic $\mathrm{C}-\mathrm{D}$ bonds during the process. The net result of the exchange reaction sequence is formation of an HD molecule. This process was found to be barrierless at low surface temperatures, while an activation barrier of $130 \mathrm{~K}$ was found for surface above

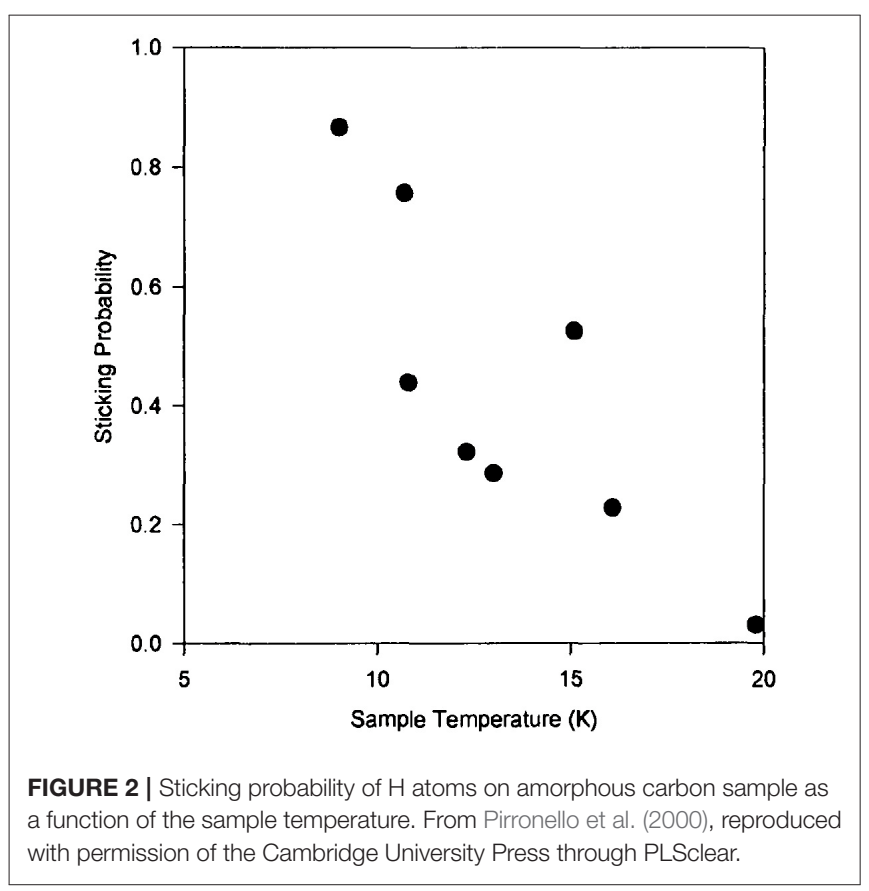

$100 \mathrm{~K}$. An abstraction cross-section of $0.03 \AA^{2}$ was evaluated for $\mathrm{H}$ atoms $(300 \mathrm{~K})$ impinging on the analog sample at room temperature. Furthermore, the nascent HD molecules formed in the aliphatic sites should not be in a highly excited state as exoenergeticity of formation reaction is reduced by $\sim 4 \mathrm{eV}$ which is necessary to break the $\mathrm{C}-\mathrm{H}$ bond. $\mathrm{H}_{2}$ formation is not specific to $\mathrm{H}$ atom chemisorbed on aliphatic carbon sites. In fact, $\mathrm{H}_{2}$ formation was observed in experiments carried out on hydrogenated aromatic carbon material with varying complexity starting from a simple polyaromatic hydrocarbon molecule (coronene film) (Mennella et al., 2012; Thrower et al., 2012) to complex carbon soots with a marked aromatic character (Mennella, 2011). In these cases, an initial carbon aromatic $\mathrm{sp}^{2}$ to aliphatic $\mathrm{sp}^{3}$ rehybridization reaction was followed by the same exchange reactions as those observed in the aliphatic carbon sample. VUV photolysis of hydrogenated amorphous carbon was suggested to be a feasible route to $\mathrm{H}_{2}$ formation (Alata et al., 2014). However, this mechanism alone can not explain the catalytic role of carbon grains and can only be a marginal source of $\mathrm{H}_{2}$ in the absence of efficient $\mathrm{H}$ atom addition process.

$\mathrm{H}_{2}$ formation involving chemisorbed $\mathrm{H}$ atoms has recently been investigated using Mg-rich hydroxylated amorphous silicates (Mennella and Suhasaria, 2019). The dust grain analogs prepared by laser ablation of an oxide mixture showed a broad infrared band resulting from chemisorbed hydroxyl groups. These hydroxylated silicate surfaces were exposed to D atoms with an energy of a few tens of meV. The catalytic $\mathrm{H}_{2}$ formation

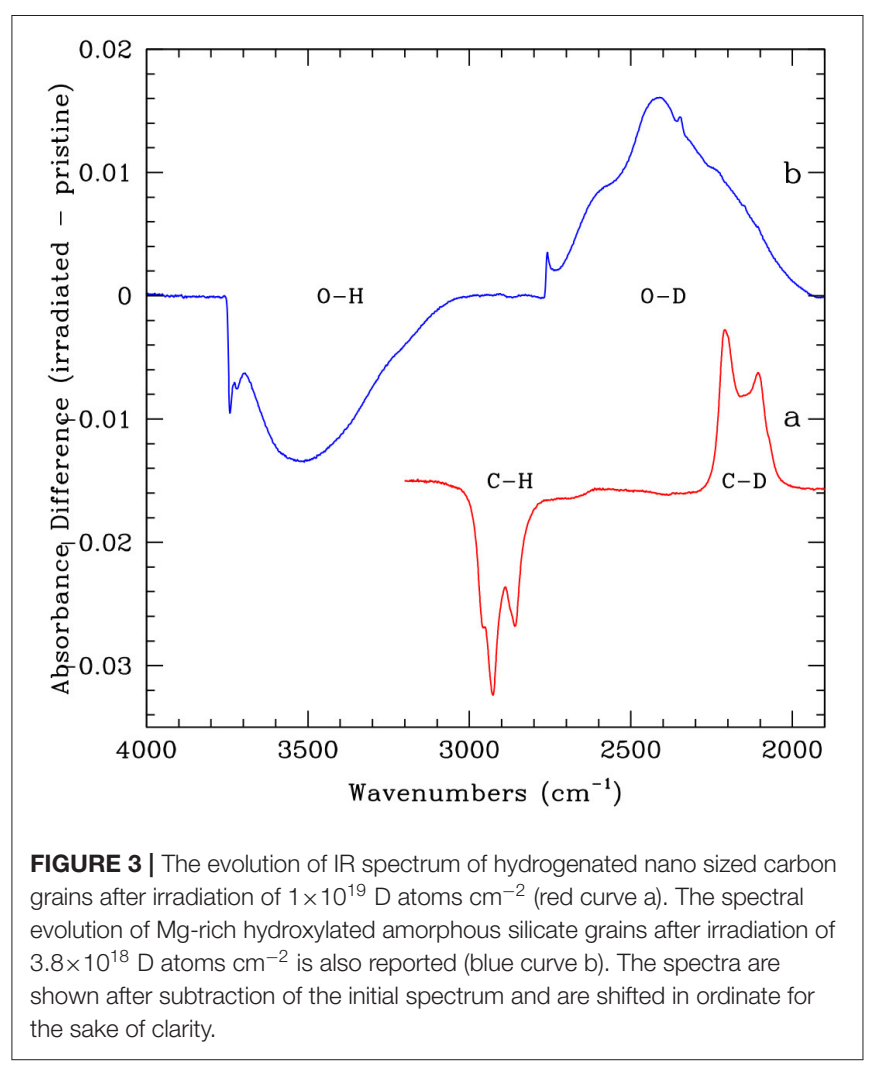


was a result of the exchange reaction similar to those on hydrogenated carbon surfaces. Figure 3 (blue curve b) shows a reduction in the number of $\mathrm{O}-\mathrm{H}$ stretching modes due to the abstraction of already bonded $\mathrm{H}$ atoms in $\mathrm{O}-\mathrm{H}$ groups of silicates. At the same time, new $\mathrm{O}-\mathrm{D}$ bonds are formed on silicate grains. In the process, $\mathrm{HD}$ molecules are formed via exchange reactions.

\section{FINAL REMARKS}

Laboratory investigations are crucial to the understanding of the physico-chemical processes involved in $\mathrm{H}_{2}$ formation. They have demonstrated how weakly adsorbed $\mathrm{H}$ atoms on refractory grain analogs at temperatures $<20 \mathrm{~K}$ migrate to another $\mathrm{H}$ atom and recombine to $\mathrm{H}_{2}$ via Langmuir-Hinshelwood mechanism. It is also evident that the ejected $\mathrm{H}_{2}$ molecules after formation on such grain analogs are likely to be vibrationally hot but rotationally cold. In the limited number of studies that concerns $\mathrm{H}_{2}$ formation involving chemisorbed $\mathrm{H}$ atoms, LH mechanism was found to be inefficient at higher grain temperature and $\mathrm{H}_{2}$ formation relies on $\mathrm{ER} / \mathrm{HK}$ mechanism. Laboratory investigations have also indicated that varying carbon based structures altering from a simple aliphatic to an aromatic to a mixed aliphatic-aromatic system catalyze $\mathrm{H}_{2}$ molecules.

\section{REFERENCES}

Alata, I., Cruz-Diaz, G. A., Caro, G. M. M., and Dartois, E. (2014). Vacuum ultraviolet photolysis of hydrogenated amorphous carbons-I. interstellar $\mathrm{H}_{2}$ and $\mathrm{CH}_{4}$ formation rates. Astron. Astrophys. 569:A119. doi: 10.1051/0004-6361/201323118

Baouche, S., Gamborg, G., Petrunin, V. V., Luntz, A. C., Baurichter, A., and Hornekær, L. (2006). High translational energy release in $\mathrm{H}_{2}\left(\mathrm{D}_{2}\right)$ associative desorption from H (D) chemisorbed on C (0001). J. Chem. Phys. 125:084712. doi: $10.1063 / 1.2220565$

Cazaux, S., and Tielens, A. G. G. M. (2004). $\mathrm{H}_{2}$ formation on grain surfaces. Astrophys. J. 604:222. doi: 10.1086/381775

Colangeli, L., Henning, T., Brucato, J. R., Clément, D., Fabian, D., Guillois, O., et al. (2003). The role of laboratory experiments in the characterisation of silicon-based cosmic material. Astron. Astrophys. Rev. 11, 97-152. doi: 10.1007/s00159-002-0017-x

Creighan, S. C., Perry, J. S. A., and Price, S. D. (2006). The rovibrational distribution of $\mathrm{H}_{2}$ and $\mathrm{HD}$ formed on a graphite surface at 15-50 K. J. Chem. Phys. 124:114701. doi: 10.1063/1.2174878

Cuppen, H. M., and Herbst, E. (2005). Monte carlo simulations of $\mathrm{H}_{2}$ formation on grains of varying surface roughness. Monthly Notices R. Astron. Soc. 361, 565-576. doi: 10.1111/j.1365-2966.2005.09189.x

Draine, B. T. (2003). Interstellar dust grains. Annu. Rev. Astron. Astrophys. 41, 241-289. doi: 10.1146/annurev.astro.41.011802.094840

Draine, B. T. (2010). Physics of the Interstellar and Intergalactic Medium. Princeton and Oxford: Princeton University Press. doi: 10.2307/j.ctvcm4hzr

Ferriere, K. M. (2001). The interstellar environment of our galaxy. Rev. Mod. Phys. 73:1031. doi: 10.1103/RevModPhys.73.1031

Gavilan, L., Lemaire, J. L., Vidali, G., Sabri, T., and Jæger, C. (2014). The formation of molecular hydrogen on silicate dust analogs: the rotational distribution. Astrophys. J. 781:79. doi: 10.1088/0004-637X/781/2/79

Gould, R. J., and Salpeter, E. E. (1963). The interstellar abundance of the hydrogen molecule. I. Basic processes.: 1963. Astrophys. J. 138:393. doi: 10.1086/147654

Hama, T., and Watanabe, N. (2013). Surface processes on interstellar amorphous solid water: adsorption, diffusion, tunneling reactions, and nuclear-spin conversion. Chem. Rev. 113, 8783-8839. doi: 10.1021/cr4000978
This occurs through an exchange reaction when $\mathrm{H}$ atoms are already pre-adsorbed on them. The role of hydroxylated amorphous silicates as catalysts for $\mathrm{H}_{2}$ formation has also been highlighted in a recent study. It is apparent that so far, most laboratory investigations have focused either on physisorption or chemisorption formation mechanism. However, the presence of $\mathrm{H}_{2}$ in a wide variety of space environments hints to the fact that the two formation mechanism are not discrete but they should be operating at the same time. Thus, there is a need for a systematic approach that underlines the intertwining of the two formation mechanisms.

\section{AUTHOR CONTRIBUTIONS}

All authors contributed to the article and approved the submitted version.

\section{ACKNOWLEDGMENTS}

We thank V. Pirronello for a careful reading of the manuscript and helpful comments and suggestions. We also thank N. Ferrigno for helping us in the editing of the manuscript. This work has been supported by Istituto Nazionale di Astrofisica (INAF).

He, J., Frank, P., and Vidali, G. (2011). Interaction of hydrogen with surfaces of silicates: single crystal vs. amorphous. Phys. Chem. Chem. Phys. 13, 15803-15809. doi: 10.1039/c1cp21601e

Henning, T. (2010). "Laboratory astrophysics of cosmic dust analogues," in Astromineralogy Vol. 815, ed T. Henning (Heidelberg; Berlin: Springer), 313329. doi: 10.1007/978-3-642-13259-9_8

Hollenbach, D., and Salpeter, E. E. (1971). Surface recombination of hydrogen molecules. Astrophys. J. 163:155. doi: 10.1086/150754

Hornekær, L., Rauls, E., Xu, W., Šljivančanin, Ž., Otero, R., Stensgaard, I., et al. (2006a). Clustering of chemisorbed H (D) atoms on the graphite (0001) surface due to preferential sticking. Phys. Rev. Lett. 97:186102. doi: 10.1103/PhysRevLett.97.186102

Hornekær, L., Šljivančanin, Ž., Xu, W., Otero, R., Rauls, E., Stensgaard, I., et al. (2006b). Metastable structures and recombination pathways for atomic hydrogen on the graphite (0001) surface. Phys. Rev. Lett. 96:156104. doi: 10.1103/PhysRevLett.96.156104

Islam, F., Latimer, E. R., and Price, S. D. (2007). The formation of vibrationally excited HD from atomic recombination on cold graphite surfaces. J. Chem. Phys. 127:064701. doi: 10.1063/1.2754684

Jäger, C., Gail, H.-P., Rietmeijer, F. J., Nuth, J. A., Mutschke, H., and Mennella, V. (2015). "Formation of nanoparticles and solids", in Laboratory Astrochemistry: From Molecules through Nanoparticles to Grains, eds S. Schlemmer, H. Mutschke, T. Giesen, and C. Jäger (Wiley), 419-500. doi: 10.1002/9783527653133.ch7

Jones, A. P., Fanciullo, L., Köhler, M., Verstraete, L., Guillet, V., Bocchio, M., et al. (2013). The evolution of amorphous hydrocarbons in the ISM: dust modelling from a new vantage point. Astron. Astrophys. 558:A62. doi: 10.1051/0004-6361/201321686

Katz, N., Furman, I., Biham, O., Pirronello, V., and Vidali, G. (1999). Molecular hydrogen formation on astrophysically relevant surfaces. Astrophys. J. 522:305. doi: $10.1086 / 307642$

Krugel, E. (2007). An Introduction to the Physics of Interstellar Dust. New york and London: Taylor \& Francis. doi: 10.1201/b15897

Latimer, E. R., Islam, F., and Price, S. D. (2008). Studies of HD formed in excited vibrational states from atomic recombination on cold graphite surfaces. Chem. Phys. Lett. 455, 174-177. doi: 10.1016/j.cplett.2008.02.105 
Lemaire, J.-L., Vidali, G., Baouche, S., Chehrouri, M., Chaabouni, H., and Mokrane, H. (2010). Competing mechanisms of molecular hydrogen formation in conditions relevant to the interstellar medium. Astrophys. J. Lett. 725:L156. doi: 10.1088/2041-8205/725/2/L156

Mennella, V. (2006). Activation energy of $\mathrm{C}-\mathrm{H}$ bond formation in carbon grains irradiated with hydrogen atoms. Astrophys. J. Lett. 647:L49. doi: $10.1086 / 507296$

Mennella, V. (2008). HD formation by abstraction of H/D chemisorbed in carbon grains with $\mathrm{D} / \mathrm{H}$ atoms under simulated interstellar conditions. Astrophys. J. Lett. 684:L25. doi: 10.1086/592001

Mennella, V. (2011). "Interaction of atomic hydrogen with carbon grains," in PAHs and the Universe, Vol. 46, eds C. Joblin and A. G. G. M.Tielens. (Cambridge: EDP Sciences), 393-398. doi: 10.1051/eas/1146040

Mennella, V., Hornekær, L., Thrower, J., and Accolla, M. (2012). The catalytic role of coronene for molecular hydrogen formation. Astrophys. J. Lett. 745:L2. doi: 10.1088/2041-8205/745/1/L2

Mennella, V., and Suhasaria, T. (2019). $\mathrm{H}_{2}$ formation on Mg-rich amorphous silicates. Int. Astron. Union. 15, 109-113. doi: 10.1017/S1743921319008834

Perets, H. B., Lederhendler, A., Biham, O., Vidali, G., Li, L., Swords, S., et al. (2007). Molecular hydrogen formation on amorphous silicates under interstellar conditions. Astrophys. J. Lett. 661:L163. doi: 10.1086/518862

Pirronello, V., Biham, O., Liu, C., Shen, L., and Vidali, G. (1997a). Efficiency of molecular hydrogen formation on silicates. Astrophys. J. Lett. 483:L131. doi: $10.1086 / 310746$

Pirronello, V., Biham, O., Manico, G., Roser, J., and Vidali, G. (2000). "Laboratory studies of molecular hydrogen formation on surfaces of astrophysical interest," in Molecular Hydrogen in Space, eds F. C. Combes and G. Pineau des Forets (Cambridge: Cambridge University Press), 71. doi: 10.1017/CBO9780511564635.012

Pirronello, V., Liu, C., Roser, J. E., and Vidali, G. (1999). Measurements of molecular hydrogen formation on carbonaceous grains. Astron. Astrophys. 344, 681-686.

Pirronello, V., Liu, C., Shen, L., and Vidali, G. (1997b). Laboratory synthesis of molecular hydrogen on surfaces of astrophysical interest. Astrophys. J. Lett. 475:L69. doi: 10.1086/310464

Thrower, J. D., Jørgensen, B., Friis, E. E., Baouche, S., Mennella, V., Luntz, A. C., et al. (2012). Experimental evidence for the formation of highly superhydrogenated polycyclic aromatic hydrocarbons through $\mathrm{H}$ atom addition and their catalytic role in $\mathrm{H}_{2}$ formation. Astrophys. J. 752:3. doi: $10.1088 / 0004-637 \mathrm{X} / 752 / 1 / 3$
Van de Hulst, H. C. (1948). Evolution and physics of solid particles. Harv. Obs. Monogr. 7:73.

Vidali, G. (2013). $\mathrm{H}_{2}$ formation on interstellar grains. Chem. Rev. 113, 8762-8782. doi: $10.1021 / \mathrm{cr} 400156 \mathrm{~b}$

Vidali, G., Li, L., Roser, J. E., and Badman, R. (2009). Catalytic activity of interstellar grains: formation of molecular hydrogen on amorphous silicates. Adv. Space Res. 43, 1291-1298. doi: 10.1016/j.asr.2008.12.019

Vidali, G., Pirronello, V., Li, L., Roser, J., Manico, G., Congiu, E., et al. (2007). Analysis of molecular hydrogen formation on low-temperature surfaces in temperature programmed desorption experiments. J. Phys. Chem. A 111, 12611-12619. doi: 10.1021/jp0760657

Wakelam, V., Bron, E., Cazaux, S., Dulieu, F., Gry, C., Guillard, P., et al. (2017) $\mathrm{H}_{2}$ formation on interstellar dust grains: the viewpoints of theory, experiments, models and observations. Mol. Astrophys. 9, 1-36. doi: 10.1016/j.molap.2017.11.001

Watanabe, N., Kimura, Y., Kouchi, A., Chigai, T., Hama, T., and Pirronello, V. (2010). Direct measurements of hydrogen atom diffusion and the spin temperature of nascent $\mathrm{H}_{2}$ molecule on amorphous solid water. Astrophys. J. Lett. 714:L233. doi: 10.1088/2041-8205/714/2/ L233

Watanabe, N., and Kouchi, A. (2008). Ice surface reactions: a key to chemical evolution in space. Prog. Surf. Sci. 83, 439-489. doi: 10.1016/j.progsurf.2008.10.001

Zecho, T., Güttler, A., Sha, X., Jackson, B., and Küppers, J. (2002a). Adsorption of hydrogen and deuterium atoms on the (0001) graphite surface. J. Chem. Phys. 117, 8486-8492. doi: 10.1063/1.1511729

Zecho, T., Güttler, A., Sha, X., Lemoine, D., Jackson, B., and Küppers, J. (2002b). Abstraction of D chemisorbed on graphite (0001) with gaseous $\mathrm{H}$ atoms. Chem. Phys. Lett. 366, 188-195. doi: 10.1016/S0009-2614(02)01573-7

Conflict of Interest: The authors declare that the research was conducted in the absence of any commercial or financial relationships that could be construed as a potential conflict of interest.

Copyright $(02021$ Suhasaria and Mennella. This is an open-access article distributed under the terms of the Creative Commons Attribution License (CC BY). The use, distribution or reproduction in other forums is permitted, provided the original author(s) and the copyright owner(s) are credited and that the original publication in this journal is cited, in accordance with accepted academic practice. No use, distribution or reproduction is permitted which does not comply with these terms. 